\title{
EEG Signals based Brain Source Localization Approaches
}

\author{
Anwar Ali Gaho ${ }^{1}$, Sayed Hyder Abbas Musavi ${ }^{2}$ \\ Department of Electrical Engineering, Faculty of \\ Engineering Science and Technology, Indus University \\ Karachi, Sindh, Pakistan
}

\author{
Munsif Ali Jatoi ${ }^{3}$ \\ Department of Electrical Engineering Technology \\ Benazir Bhutto Shaheed University of Technology and Skill \\ Development, Khairpur, Sindh, Pakistan
}

\author{
Muhammad Shafiq ${ }^{4}$ \\ University of Innsbruck, IMGI, Austria \\ SUPARCO, Karachi, Pakistan
}

\begin{abstract}
This article is focused on the overview of functionality of the neurons and investigation of the current research and algorithms used for brain source localization. The human brain is made up of active neurons and continuously generates electrical impulses on scalp surface. The neurons transmit the message through the dendrites called pyramidal cells. The active parts of the brain are addressed and measured by various neuroimaging techniques such as electroencephalography (EEG), magnetoencephalography (MEG) etc. These techniques help to diagnose pathological, physiological, mental and functional abnormalities of the brain. EEG is a high temporal resolution and a low spatial resolution technique which yields the non-invasively potential difference measurements between pair of electrodes over the scalp. It is used in understanding behavior of brain which is further used to analyze various brain disorders. EEG brain source localization has remained an active area of research in neurophysiology since last couple of decades and still being investigated in terms of its processing time, resolution, localization error, free energy, integrated techniques and algorithms applied. In this paper, several approaches of forward problem, inverse problem and Bayesian framework have been explored to address the uncertainties and issues of localization of the neural activities incurring in the brain.
\end{abstract}

Keywords-Electroencephalograph; brain source localization; forward problem; inverse problem; bayesian framework

\section{INTRODUCTION}

The human brain is made of $10^{10}$ neurons. The neurons pass out the message through the dendrites called pyramidal cells so called neural activity. This brain neural activity causes the generation of the electrical potentials over the scalp[1,2]. The neurons' main function is to communicate chemically and electrically with other neurons[3]. They send the messages from one lobe to another lobe thereby causing the dipole currents inside the brain generated due to the stimuli or other brain disorders. and subsequently generate the synapse, presynaptic and post-synaptic actions in the brain [4]. This section consists of the following parts:

\section{A. Brain Neurons}

The brain controls all main functions of the body. It is composed of cerebrum, cerebellum, and brainstem[5, 6]. The cerebrum is divided into left and right hemispheres. The fissures or grooves, divides cerebrum into four main parts i.e., frontal, temporal lobe, parietal lobe and occipital lobe. The mid-brain, pons, and medulla are interconnected with cerebrum with spinal cord[7]. Since the neuron is composed of cell body, nucleus, axons, nodes of ranvier, myelin sheath and dendrites as shown Fig.1. The neuron's structure physically change with the passage of the time and age of the subjects such as from infant to old [8].

\section{B. EEG Current Dipoles}

On the application of the stimuli, electricity is generated by a particular group of neurons in the cortex so called dipoles as shown in Fig. 2. This dipole generates the electric field and creates potential field which is picked up by the scalp EEG electrodes.

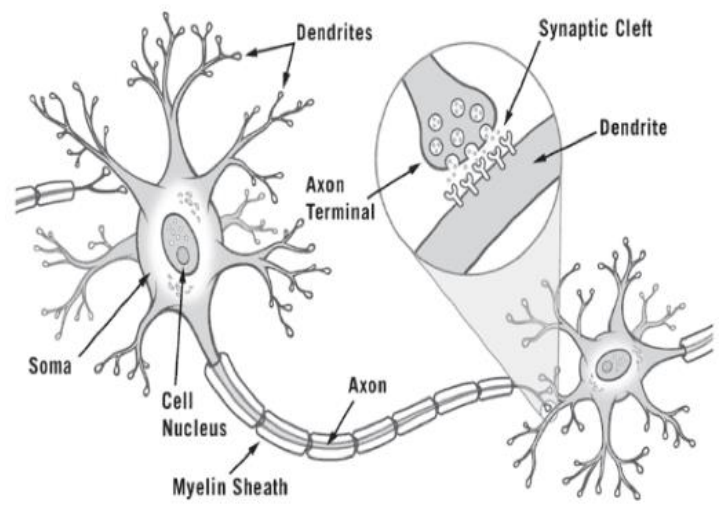

Fig. 1. Neuron structure[3]

EEG measures the current caused by the excitations of the dendrites of pyramidal neurons. The potential differences are caused by summed postsynaptic potentials from pyramidal cells that create diploes between soma and apical dendrites as shown in Fig. 3. 


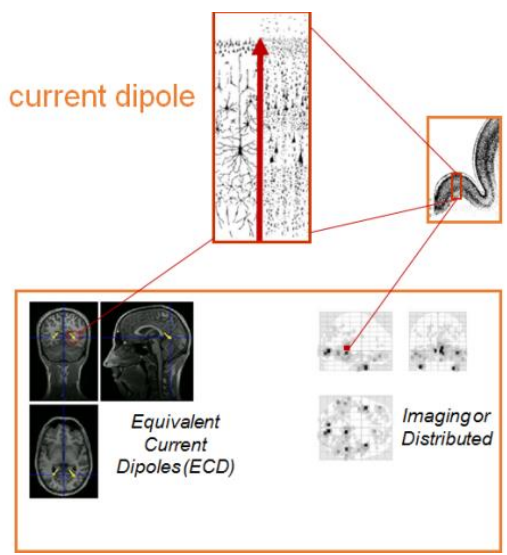

Fig. 2. Generation of current dipoles[6]

\section{EEG Generators}

The pre-synaptic and post-synaptic are linked through the synapses. The neurons process the inhibitory action potentials and then convert it into post synaptic potentials for firing other neurons as shown in Fig. 3. The signals are superimposed synchronized and pass from grey matter and become normal to cortical surface thereby creating potentials on cortical surface.

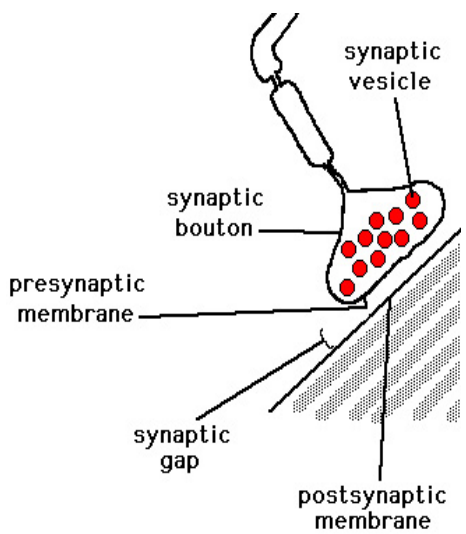

Fig. 3. Releasing ions [9]

The depolarization or excitatory postsynaptic potential (EPSP) reduces to $-40 \mathrm{mV}$ in the intracellular volume. The hyper polarization or inhibitory postsynaptic potential (IPSP) potential difference is subsequently increased. Due to the action of $\mathrm{Na}+\mathrm{K}+$ and $\mathrm{Cl}$ - in the brain, potential difference of $70-110 \mathrm{mV}$ for the period of 0.3 millisecond is generated as shown the Fig. 4.

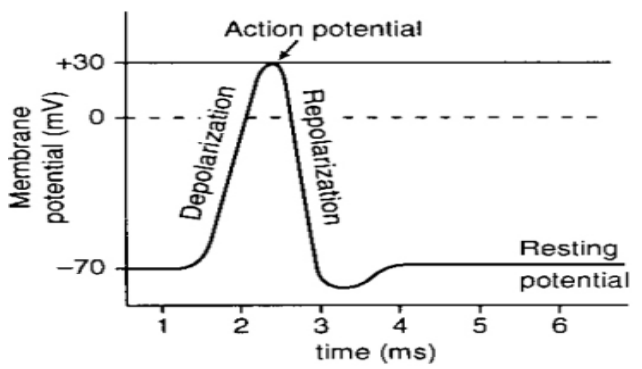

Fig. 4. Action potential [10]

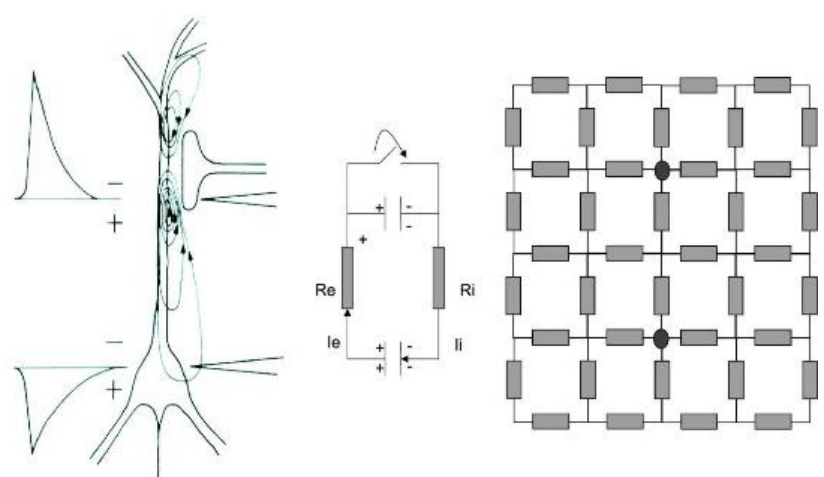

Fig. 5. Equivalent circuits for a neuron [12]

An equivalent circuit of capacitor, resistor and potential source is shown in Fig. 5. At rest, voltage stored between the intra-cellular and extracellular areas is denoted by two charged capacitors. One is assigned for potential difference between apical dendrites side and other for potential difference at the cell body for basal dendrite side. Action of charging and discharging takes place between capacitors with the on and off switch.

\section{EEG Applications}

EEG being a revolutionized neuroimaging technique is not only used for helping in diagnosing pathological, physiological, mental and functional abnormalities of the brain but also for understanding epilepsy, schizophrenia, and Alzheimer's diseases. EEG is assisting in diagnosing disabilities, hyperactivity, sleep, awakening disorders, depression and surgery in patient with seizures. Moreover, EEG is useful for identifying coma, brain and death problems and locating injury, stroke, tumor, evoked potentials sources and brain cognitive behavior. EEG is also extensively used in investigating drug effects, aesthesia issues, brain growth and death. It is currently being integrated with other brain imaging modalities for better human life [11].

In the literature, there are several other neuroimaging techniques such as MEG, ECoG. Functional MRI (fMRI) and NIRS used in brain neurology. Their comparative analysis is given in TABLE I. where EEG mostly seems to be most favorable neuroimaging technique in given characteristics such as measurement, resolution and method.

TABLE I. COMPARISONS OF NEUROIMAGING TECHNIQUES[11]

\begin{tabular}{|l|l|l|l|l|}
\hline Technique & Measurement & Temporal & Spatial & Method \\
\hline Type & Type & $(\boldsymbol{m}$ sec $)$ & $(\boldsymbol{m m})$ & Type \\
\hline EEG & Electrical & 0.05 & 10 & Invasive \\
\hline MEG & Magnetic & 0.05 & 5 & Invasive \\
\hline ECoG & Electrical & 0.003 & 1 & Invasive \\
\hline fMRI & Metabolic & 1 & 1 & Non-Invasive \\
\hline NIRS & Metabolic & 1 & 5 & Non-Invasive \\
\hline
\end{tabular}

This article is divided into three sessions. Session I is about the introduction of EEG source localization. Section II covers the EEG brain source localization approaches. Section III gives conclusion and remarks. 


\section{EEG SOURCE LOCALIZATION ALGORITHIMS}

The forward and inverse problems are used for locating the sources of the brain activity. The former problem measures potentials over the scalp of the skull whereas the later yields sources of the activity and localization[12]. Both Techniques are discussed in details in subsequent sections.

\section{A. Forward Problem}

Forward problem is related to measuring of the potentials with the electrodes from the surface of the scalp and has a unique solution as shown in Fig. 6.

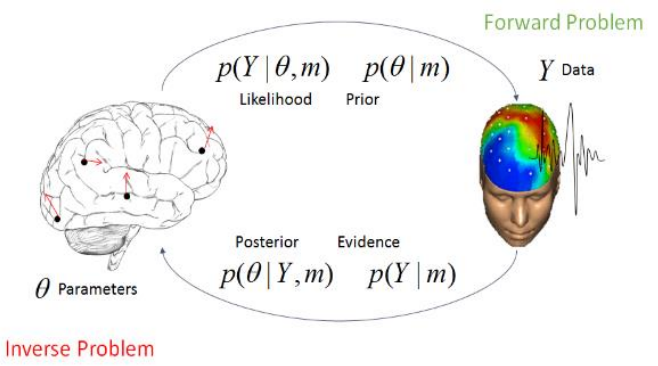

Fig. 6. Forward problem [12]

The grey matter as shown in Fig. 9. is composed of pyramidal cells which forward the currents to scalp surface through the apical dendrites with 2 milli-second duration. Orientation of the neurons causes the superimposing and cancelling out the electrical signals. Since neural activity is neither the static and nor the dynamic and therefore it is called quasi- statics conditions [13]. The Poisson's equation containing the divergence operator relates the potential difference, and current densities of the volume of the brain i.e. $\nabla . J=\lim _{G \rightarrow 0} \int_{\partial G} J d S$

This integral model represents the complete spherical shape of the surface and volume $(\mathrm{G})$ where the flux in is negative and flux out is positive. Its current density form is represented by $\nabla . J=I_{m}$. Lines represent the flux caused by the currents and potentials generated in the volume[14] as shown in Fig. 7.

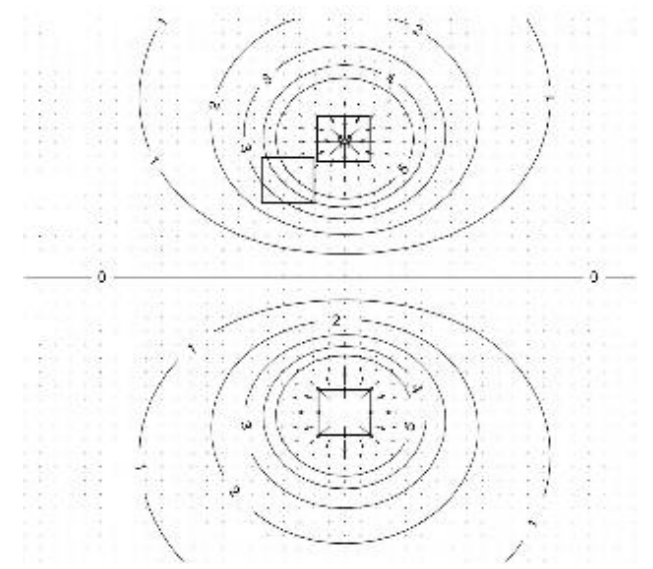

Fig. 7. Current density with equip-potential lines[12, 15]
The human head is composed of the isotropic and anisotropic tissues. The conductivity causes electric currents. The skull is made of spongiform layer, skull and scalp and composed of biological and chemical tissues [16] which affects the current flow from cortical surface to scalp. [17]. The skull consists of three layers such as a spongiform layer and two hard layers as shown in Fig.8. The conductivity tangential to the skull surface is 10 times larger than the radial conductivity.
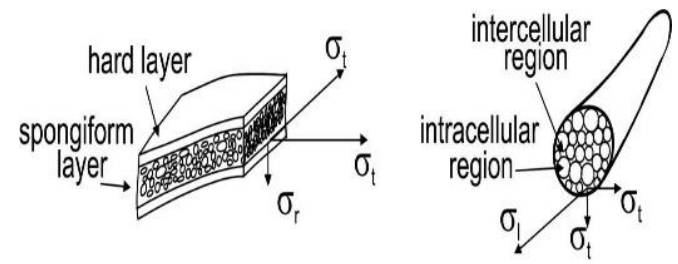

Fig. 8. Brain conductivity tissues [18].

The white matter has 9 times greater conductively than grey matter due to structure of the cells. The watery tissues have a greater conductivity. The several models of isotropic and anisotropic conductivities are incorporated in the forward problem to address the issues of the conductivities.

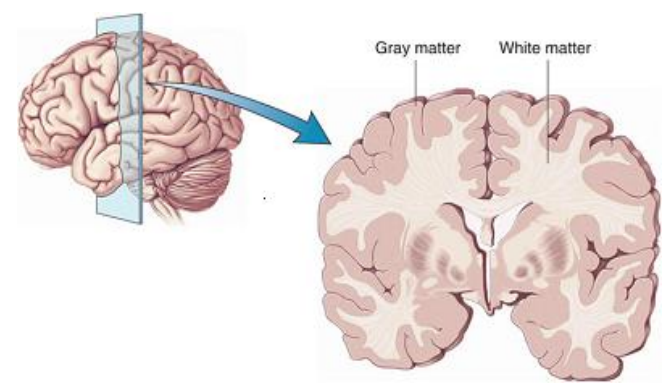

Fig. 9. Grey and white matter[7, 9]

Faraday's law gives zero results due to quasi-static conditions that is $\nabla \times \mathrm{E}=0$. Potential and electric fields with the gradient operator is given as $\mathrm{E}=-\nabla V$. The negative sign reflects electric field direction. The human head is composed of the different types of the layers. As the outer layers of the human are scalp and air. As there is no current that flows in the outer layers due to air which is non-conducting material. Therefore, the current density inside the outer air layer is zero. The current dipole of pyramid cells has the same current flown in and flow out. The dipole has six parameters including three translational parameters and three spherical parameters with a unit vector at a pint in the source space between two monopoles as shown in Fig. 10.

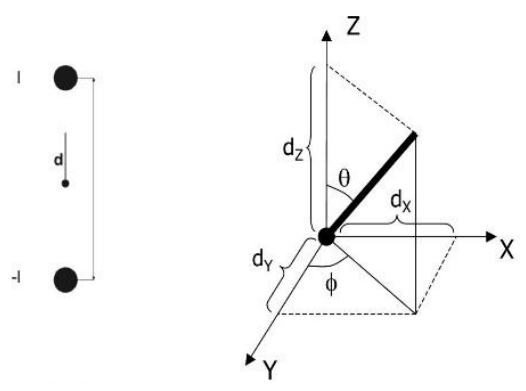

Fig. 10. Dipole parameters [12] 


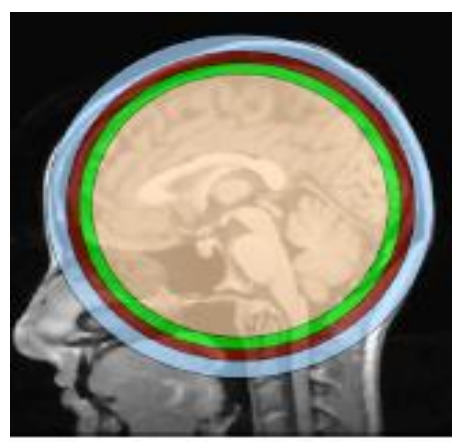

Fig. 11. Simple head model [9]

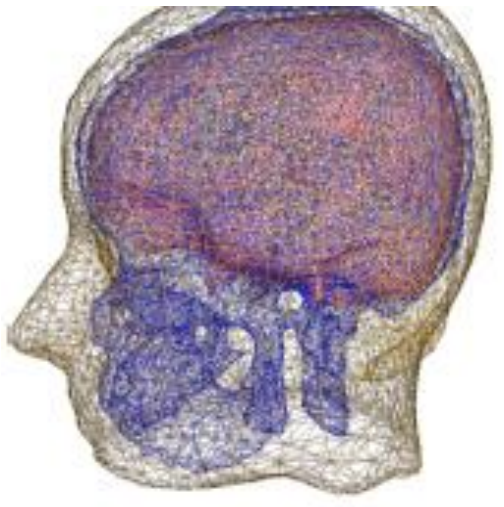

Fig. 12. Realistic head model[24]

The human head is a sphere and is composed of the conductivity tissues of skull and scalp. It is made of three concentric circles representing each circle as a layer [18]. Its conductivities are modeled in terms of radial and tangential layers[19]. The prolate and oblate spheroids or eccentric spheres are modeled and discussed in the literature. The Berg approximation [20] represents a single-sphere model for a three to four layer sphere model for increasing the computation and geometry accurateness [21,22]. Simple head models are single layer spheroid models which are simple, fast and accurate one. The realistic head models with complexity are more numerical and realistic such as boundary element method (BEM)[19] and finite element method (FEM) [14, 23].

The realistic head models also called numerical models (Fig. 12) in computational time are higher than multi-layer spheres or analytical models (Fig. 11) due to formation of thousand pieces [25]. A good approach between spherical head model and realistic head one is counted a good quality of sensor-fitted approach i.e. exact on the location of the activity[26].

The BEM numerical technique calculates the surface potentials produced by current sources located in a piecewise uniform volume formed with isotropic conductivities structures $[9,27]$. The pieces are made in such size that they are so small for yielding isotropic conductivities[28]. There are three boundaries with the three layers in spherical model. Each layer is put into pieces and is computed with small boundary elements. The distances of the layers from brain, skull and scalp to the center positions are $8 \mathrm{~cm}, 8.5 \mathrm{~cm}$ and 9.2 $\mathrm{cm}$, respectively[29]. Each layer is digitized in triangles, for computing the potential at its center (see Fig 13 and 14).

The Green's first identity for integration is implemented to solve Poisson's equation in a realistic head model is the finite element method (FEM). The 3D volume conductor is digitized into small elements as shown in Fig 13 and Fig 14.
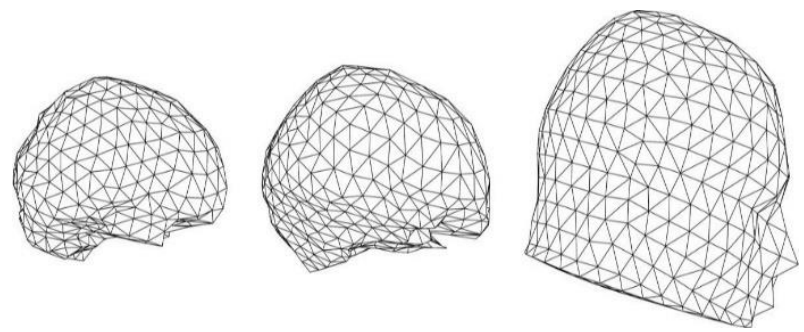

Fig. 13. Meshes of human head[14]

Fig 13, illustrates 2D volume conductor digitized with triangles and Fig.14 states the mesh in 2D coronal slice view only. In summary, forward problem for brain source localization covers all the algorithms and theory related to scalp, conductive medium and measuring potential differences techniques.

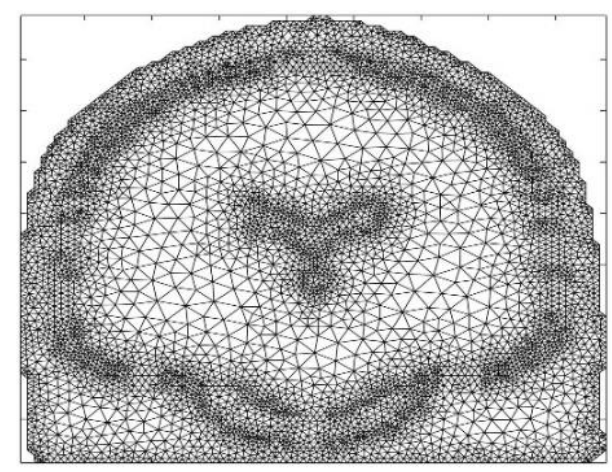

Fig. 14. Mesh in 2D coronal slice[12, 15]

\section{B. Inverse Problem}

The inverse problem is an ill-posed and non-unique problem due to a reason that there are infinite dipoles inside the cortical conductor and limited number of the electrodes over scalp for fitting the data causing the uncertainties. Generally, true model in inversion problem consists of estimation problem and appraisal problem [15, 30] (see Fig $15)$.

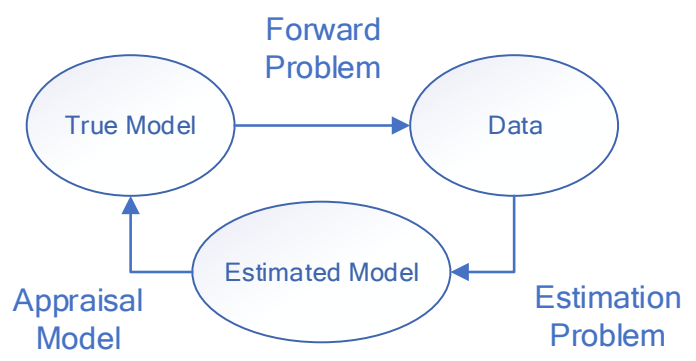

Fig. 15. Inversion problem of physical model [46] 
Two methodologies such as equivalent current dipole (ECD) for limited areas for underlying neural activities and linear distributed for whole volume are assumed for current source densities. [31,32]. Several algorithms have been developed with a minimum of localization error and high resolution and less computational time. The hierarchy of the inverse solutions and their history are shown in Fig.16 and Fig. 17. respectively.

The minimum norm estimation (MNE) estimates sources with $1 \mathrm{~cm}$ resolution [15]. It yields predicted information and provides better localization with increasing the number of magnetometers. It gives good resolution and current estimation. It provides improved localization error.

The low-resolution electromagnetic tomography (LORETA) estimates all active, neighboring and boundary areas. It not only yields good time resolution but also provides the poor spatial resolution of the blurring images. Further, it yields less errors and used for depth sources. LORETA and its family use the current source density for reconstruction.

The focal underdetermined system solution (FOCUSS) is a tomographic reconstruction scheme. It is high resolution nonparametric technique. It has a good spatial resolution. FOCUSS is used for deep source localizations. Recursive Multiple Signal Classification (Recursive MUSIC) [33] is a modified form of a RAP MUSIC. It yields less error of localization and is easy for processing of computation. It can face the situations where there are constraints or limits.

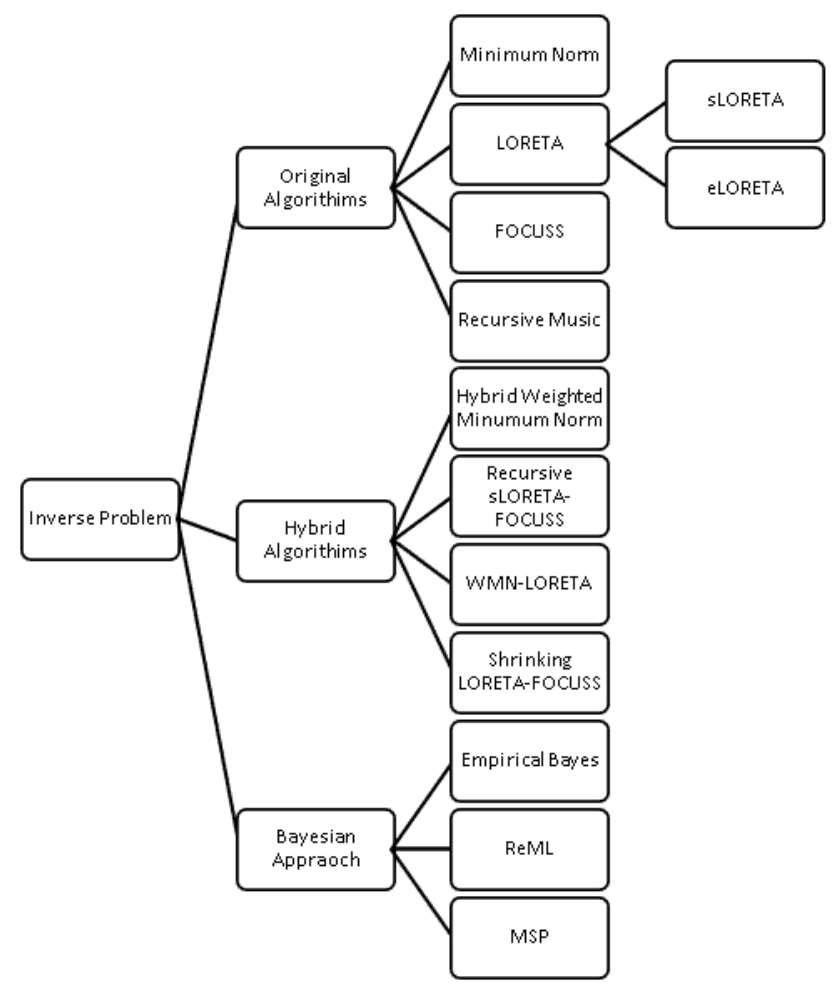

Fig. 16. Solutions of inverse problem [15]
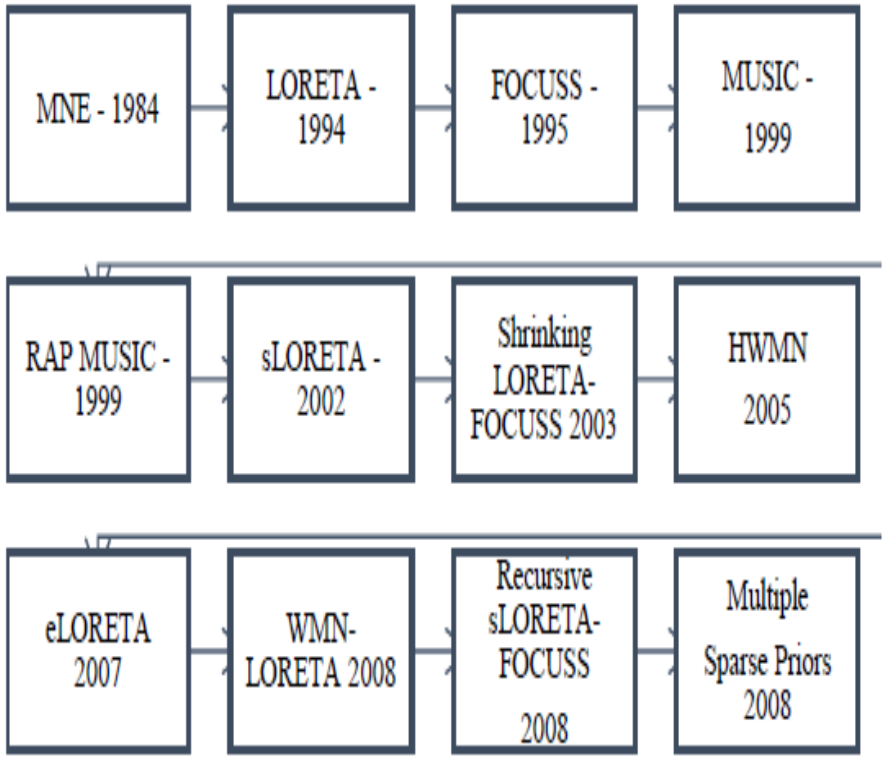

Fig. 17. History of solutions of the inverse problem [15]

The hybrid weighted minimum norm (HWMN) [15] is the modified form of LORETA [34, 39], FOCUSS [35] and WMN [15, 36], It yields the smoothness with a long processing time and therefore losses data while computing. The standard LORETA (sLORETA) [40] yields uniform variance across the whole brain volume and yields zero localization error. The results are verified on experimenting on 6430 voxels with having $5 \mathrm{~mm}$ spatial resolution. It has an exact localization with a zero error. Exact LORETA (eLORETA) [25] yields the variance of unity. All its simulations are based on LORETA software where zero-bias creates zero-localization error. Its weight matrix yields correct value with minimized error (12 to $7 \mathrm{~mm}$ ). It has also the access of the touch to deep sources. There are some comparative studies for sLORETA and eLORETA[37]. WMN-LORETA experiments are carried out through by both simulation and experimented basis with 138 electrodes. Firstly, resolution matrix is designed. In these experiments such as conditions of identity matrix are achieved. However, error is subsequently reduced and accuracy is enhanced with less execution time. In Recursive sLORETA-FOCUSS, sLORETA gives smoothness whereas FOCUSS gives sparse solution and increase localization error with $494.03 \mathrm{~mm}$ when experiments are conducted. Its processing time is reduced to 330.45. Shrinking LORETA-FOCUSS a derived from LORETA and FOCUSS. It has the complexity of the weight matrix and iteration performance due to which it takes a time for processing. It provides good smoothness with low localization error. The sources construction with high spatial resolution is achieved. It uses LORETA and current densities for smoothness thereby subsequently weight matrix is computed. Summary of the inverse methods and their comparison are given in Table II and Table III. 
TABLE II. SUMMARY OF INVERSE METHODS[10, 15]

\begin{tabular}{|c|c|}
\hline Method & Characteristics/Comparison \\
\hline MNE[15, 38] & $\begin{array}{l}\text { - It has good resolution and current estimation and } \\
\text { yields good localization error as compared to } \\
\text { LORETA, WMN etc. } \\
\text { - It fails for deep source localization and is } \\
\text { incapable of localizing non-boundary sources. }\end{array}$ \\
\hline $\begin{array}{l}\text { LORETA } \\
{[39]}\end{array}$ & $\begin{array}{l}\text { - It is popular due to source localization and } \\
\text { citations. } \\
\text { - However it is incapable of improving low spatial } \\
\text { localization. }\end{array}$ \\
\hline sLORETA [40] & $\begin{array}{l}\text { It yields poor stability and blurriness in the } \\
\text { mage. } \\
\text { - It is a derived from LORETA and yields better } \\
\text { localization. } \\
\text { - It has poor resolution and poor performance for } \\
\text { recovering multiple sources }\end{array}$ \\
\hline eLORETA[25] & $\begin{array}{l}\text { - It is derived method of LORETA and gives } \\
\text { authentic results in localization and handles the } \\
\text { issue of variance very well. } \\
\text { - Its low resolution and blurring in images } \\
\text { increases. }\end{array}$ \\
\hline FOCUSS[35] & $\begin{array}{l}\text { Its possibility of sources localizing is on edge } \\
\text { and deep areas. } \\
\text { - It yields spatial variations in good quantity. } \\
\text { - It is crucial to bring out the features of the brain } \\
\text { activity due to its low resolutions and } \\
\text { blurredness. }\end{array}$ \\
\hline HWMN[15] & $\begin{array}{l}\text { - It is a derived from MN and LORETA and } \\
\text { provides better estimation. } \\
\text { - It uses LORETA and WMN for better } \\
\text { estimation. } \\
\text { It takes more iterations for more accurate, deep } \\
\text { localization and less errors. } \\
\text { It takes a large processing time.and these is a } \\
\text { possibility of the loss of data due to continuous } \\
\text { iterations and weight matrix involvement. }\end{array}$ \\
\hline $\begin{array}{l}\text { shrinking } \\
\text { LORETA- } \\
\text { FOCUSS[41] }\end{array}$ & $\begin{array}{l}\text { - It is derived from LORETA and FOCUSS. } \\
\text { - Its results are not experimentally validated. } \\
\text { - It provides better minimized localization error. }\end{array}$ \\
\hline MUSIC[33] & $\begin{array}{l}\text { It yields less localization error. } \\
\text { However, it takes a large processing time but } \\
\text { there is a chance of the loss of the data. }\end{array}$ \\
\hline $\begin{array}{l}\text { RAP-MUSIC } \\
{[42]}\end{array}$ & $\begin{array}{l}\text { It yields good localization error but it takes a } \\
\text { large processing time. } \\
\text { - There are chances of the loss of data. } \\
\text { - However, its random error and noise problem } \\
\text { increase the issues of its processing. }\end{array}$ \\
\hline $\begin{array}{l}\text { WMN- } \\
\text { LORETA [15] }\end{array}$ & $\begin{array}{l}\text { - It yields better resolution if compared with } \\
\text { LORETA and WMN separately. }\end{array}$ \\
\hline $\begin{array}{l}\text { Recursive } \\
\text { sLORETA- } \\
\text { FOCUSS [15] }\end{array}$ & $\begin{array}{l}\text { It is an efficient in terms of processing and less } \\
\text { localization error. }\end{array}$ \\
\hline
\end{tabular}

TABLE III. COMPARISONS OF INVERSE METHODS[15]

\begin{tabular}{|l|l|l|l|}
\hline Method & Resolution & Time & Validation \\
\hline MNE & low & high & experiment \\
\hline LORETA & low & high & experiment \\
\hline FOCUSS & low & low & experiment \\
\hline Recursive MUSIC & good & low & simulation \\
\hline sLORETA & low & high & experiment \\
\hline Shrinking LORETA-FOCUSS & low & low & simulation \\
\hline Hybrid Weighted MN & low & low & simulation \\
\hline eLORETA & low & high & experiment \\
\hline WMN-LORETA & low & low & simulation \\
\hline RecursivesLORETA-FOCUSS & low & high & simulation \\
\hline
\end{tabular}

LORETA yields good localization error and estimated current density whereas the Shrinking LORETA- FOCUSS yields energy error, localization and maximum energy error. sLORETA- FOCUSS provides excellent localization error in simulations whereas recursive sLORETA-FOCUSS provides better processing time, accuracy and more accurate results. WMN-LORETA is a best method in terms of time processing and resolution.

This was the summary of the approaches and their comparative analysis used for ill-posed inverse problem.

\section{Bayesian Approaches}

This approach has a vital role in source localization of the brain. Inversion scheme primarily consists of Bayesian approach. Much efforts are made to put the theoretical and mathematical approaches into empirical and experimental practices to locate sources of the underneath neural activities[43]. The optimized estimations are based on posterior and priori procedures called the Bayesian approach. EEG source localization depends on the estimation of priors or patches selected from parametric Bayes (PEB) so called empirical Bayes[44]. PEBs are simple parametric hierarchical linear models and parametric assumptions involve the randomly addition of Gaussian noise at each level[45]. The source space basically generates the data. The background of using this function is to limit on log-evidence or Likelihood by using Gaussian process priors on different models so called priors or covariance components of given measurements and best model automatically is selected from the hyperparameters by enforcing the conditional variances to minimum value [46]. The quality of using free energy and restricted maximum likelihood (ReML) are to get the grip on covariance components and hyperparameters [45]. These methods form an inversion scheme which helps in selection of the optimized priors either from sparse priors or distributed priors provided the nature of the data obtained. There are the definitely benefits for using inversion scheme such as it is fast and consists of the linear models for both said priors [47]. The empirical Bayes provide the best quantifying and relative information of spatial priors and accommodate multiple priors for the provision of more accurate source of the reconstruction of neural activity [48]. In empirical Bayes, different combinations of the priors so called sets of the models, are selected through the method of the model selection. The best utilization of ReML as log-likelihood is to bring improperness in using parameters of the hyperparameters [49]. Selected model is defined in terms of the covariance components.

Inversion scheme [50] is applied to the evoked highlighted neural activity by applying free energy bound or log-evidence of marginal likelihood or ReML objective function. The benefit of using ReML is that optimization can be carried out by selecting a specific model of covariance components from measurement of data irrespective of increasing the size and number of the resources. Empirical priors are the source estimated priors which can be observed during the desired pattern of the brain neural activity observed through EEG responses[51]. Its mathematical form is described as 
$Q=\sum_{i=1}^{N_{q}} h_{i} C_{i}$ where priors are basically the components, partitions or the models which have large variances so called hyperparameters which are further spitted-up into a small group of the patterns called covariance components [52]. The evidences of the highly active regions of the neural activity with the local and compact support can be modelled as source priors. In Bayesian approach, the prior source covariance which are counted as weighted sum of multiple prior components are called as empirical Bayes as available in the literature as priors for source reconstruction[53]. The highly optimized combination of the compact and desired higher neural activity priors of the desired regions can be evaluated through evidences such as using Bayesian model approach. Hyperparameters have large variances. The variety of EEG responses can be observed though EEG responses. Such extreme situations can be addressed through minimum norm constraint and ARD solutions. The Bayesian model approach provides the log-evidences and Greedy search[54, 55] is iterated on evident provided patters or components for efficiently splitting the highest hyperparameters of the highest variances into two or more new components or partitions so called models[45]. Such iteration is repeated until the evidences are stopped for increasing and optimized results are obtained[56]. In a nutshell, both the greedy search and ARD schemes are used for optimizing the neural activity of EEG source localization.

In parametric empirical Bayes (PEB) [57], several models of the source neural activities are estimated. These are experimentally or empirically selected from the different regions of the brain. The empirical priors on the sources are given by $[58,59]$ : There is a variety of picking up the priori models of neural activity. The closed and compact neural activity priors are particularly paid attention These are also known as model correlated sources[60]. Any groups of priors can be enhanced or compared with one another's evidences so called Bayesian model comparison.

A greedy search (GS) that splits highest variance or hyperparameter into more than two components and subsequently optimized parameters. This action is iterated until its evidence stops increasing [49]. In Automatic relevance determination (ARD) one can start with one component per pattern and use ARD to eliminate other unused and unnecessary patterns. Inversion Scheme [50] is comprised of MN, IID, LOR and other MSPs. Specific designed $\mathrm{M}$ weight matrix which is also called mixture matrix as covariance components are used for optimization of estimating the reconstruction of the neural activity with the current density. In this scheme at sensors levels and noise levels optimized techniques are used for maximizing the current density [61]. Minimum norm (MN) Model is represented by $Q^{\varepsilon}=I$. This model asserts that all sources are active, with equal a priori probability and that none are correlated $[50,57]$. Coherence $(\mathrm{COH})$ model is a realistic model with two components modelling independent and coherent sources respectively. Multiple sparse prior model is represented by components such as $Q^{\varepsilon}=\left\{q_{1} q_{1}^{T}, \ldots, q_{N} q_{N}^{T}\right\}$ modeling activity in $\mathrm{N}$ patterns[57, 62].

\section{CONCLUSION AND REMARKS}

In this article, several approaches of forward problem, inverse problem and Bayesian framework have been explored and discussed for EEG brain source localization in terms of their computational time, localization error, energy error, resolution etc. An EEG brain source localization not only yields the complete solution of understanding brain abnormalities, mental diseases and cognitive brain actions but it also provides the best spatiotemporal solution of diagnosing the disorders of the brain such as epilepsy and tumors etc. The forward yields only potential measurements on the scalp which are calculated through the electrodes and efforts are made to remove the noises from the signals due to uncertainties of the three layers or mediums, coupling of the cortical surfaces and environmental noises or stimuli and electrodes used for experimental purposes. EEG minimum norm and listed LORETA family algorithms used for ill-posed inverse problem subsequently yields the good results in terms of resolution localization error and computational time of the active brain sources. Bayesian framework involves the optimized selection of the patches of the surface or volume of the cortical surface or head. Multiple sparse priors (MSP) algorithm-based Bayesian algorithm is targeted for optimization of the source localization. ReML, Greedy search, ARD and Coherence approaches are used as optimization techniques in terms of giving out the good results for the maximum free energy. The uncertainties such as unnecessary active regions or patches, unnecessary equivalent current dipoles or unnecessary coupling of neighbor regions of the brain are surely removed to reach out at the exact active brain region for giving a maximum energy as discussed in Bayesian framework. However, there still more work is required on solving the forward problem and ill-posed inverse problems. There is a need of using exact analytical or numeral head models required. Conductivities and noise issues are yet to be improved by using the new techniques or algorithms of computational methods. The new integrated neuroimaging techniques with robust algorithms are yet to be worked out for giving better results in terms of spatiotemporal resolution, localization error, less processing time or even free energy. The multiple source prior technique seems to one of most advanced techniques which is capable for finding the most optimized source localization by removing unnecessary patches or their repetition.

This was an entire overview of EEG signals-based source localization. Future study will deeply be extended to cover their latest research and mathematical background which will surely will cover all the issues in optimizing the results of the brain source localization using EEG signals.

\section{REFERENCES}

[1] Q. Tran, T. Le, C. Le, and T. V. Van, "Electrophysiological modeling in generalized epilepsy using surface EEG and anatomical brain structures," in International Conference on the Development of Biomedical Engineering in Vietnam, 2017, pp. 699-704: Springer.

[2] A. Majkowski, Ł. Oskwarek, M. Kołodziej, and R. J. Rak, "An attempt to localize brain electrical activity sources using EEG with limited 
number of electrodes," Biocybernetics and Biomedical Engineering, vol. 36, no. 4, pp. 686-696, 2016.

[3] R. Cook, G. Bird, C. Catmur, C. Press, and C. Heyes, "Mirror neurons: from origin to function," Behavioral and Brain Sciences, vol. 37, no. 2, pp. 177-192, 2014.

[4] M. A. Lancaster et al., "Cerebral organoids model human brain development and microcephaly," Nature, vol. 501, no. 7467, p. 373, 2013.

[5] M. Susta, H. Papezova, S. Petranek, and K. Sonka, "Brain activation sequences," Neuroendocrinology Letters, vol. 36, no. 8, 2015.

[6] P. Milz, K. Oberauer, P. D. D. C. Kiper, and D. Lehmann, "Brain Electric Mechanisms of Modalities of Thinking," Universität Zürich, 2016.

[7] M. J. Hawrylycz et al., "An anatomically comprehensive atlas of the adult human brain transcriptome," Nature, vol. 489 , no. 7416 , p. 391 , 2012.

[8] L. G. Kiloh, A. J. McComas, and J. W. Osselton, Clinical electroencephalography. Butterworth-Heinemann, 2013.

[9] J. K. Mai, M. Majtanik, and G. Paxinos, Atlas of the human brain. Academic Press, 2015.

[10] W. Khalifa, A. Salem, M. Roushdy, and K. Revett, "A survey of EEG based user authentication schemes," in Informatics and Systems (INFOS), 2012 8th International Conference on, 2012, pp. BIO-55-BIO60: IEEE.

[11] A. Chaturvedi, "Analysing the Use of EEG, fMRI, PET and Behavioural Studies of Brain Lesioned Patients Associated with Non-Literal Language eg Metaphors, Sarcasm, Some Types of Humour Etc," 2017.

[12] Hallez, Hans, et al. "Review on solving the forward problem in EEG source analysis." Journal of neuroengineering and rehabilitation 4.1 (2007): 46.

[13] R. Plonsey and D. B. Heppner, "Considerations of quasi-stationarity in electrophysiological systems," The Bulletin of mathematical biophysics, vol. 29, no. 4, pp. 657-664, 1967.

[14] M. R. Lakshmi, D. T. Prasad, and D. V. C. Prakash, "Survey on EEG signal processing methods," International Journal of Advanced Research in Computer Science and Software Engineering, vol. 4, no. 1, 2014.

[15] M. A. Jatoi, N. Kamel, A. S. Malik, I. Faye, and T. Begum, "A survey of methods used for source localization using EEG signals," Biomedical Signal Processing and Control, vol. 11, pp. 42-52, 2014.

[16] C. H. Wolters, "Influence of tissue conductivity inhomogeneity and anisotropy on EEG/MEG based source localization in the human brain," Max Planck Institute of Cognitive Neuroscience Leipzig, 2003.

[17] C. H. Wolters, A. Anwander, X. Tricoche, D. Weinstein, M. A. Koch, and R. MacLeod, "Influence of tissue conductivity anisotropy on EEG/MEG field and return current computation in a realistic head model: a simulation and visualization study using high-resolution finite element modeling," NeuroImage, vol. 30, no. 3, pp. 813-826, 2006.

[18] V. E. Montes Restrepo, "Determination of anisotropic ratio of the skull for EEG source localization in patients with epilepsy," in 11e FirW PhD Symposium, 2010, pp. 62-62: Universiteit Gent. Faculteit Ingenieurswetenschappen.

[19] M. A. Jatoi et al., "EEG-based brain source localization using visual stimuli," vol. 26, no. 1, pp. 55-64, 2016.

[20] P. Berg and M. Scherg, "A fast method for forward computation of multiple-shell spherical head models," Electroencephalography and clinical neurophysiology, vol. 90, no. 1, pp. 58-64, 1994.

[21] B. Cuffin, "Effects of local variations in skull and scalp thickness on EEG's and MEG's," IEEE Transactions on Biomedical Engineering, vol. 40, no. 1, pp. 42-48, 1993.

[22] N. Chauveau, X. Franceries, B. Doyon, B. Rigaud, J. P. Morucci, and P. Celsis, "Effects of skull thickness, anisotropy, and inhomogeneity on forward EEG/ERP computations using a spherical three-dimensional resistor mesh model," Human brain mapping, vol. 21, no. 2, pp. 86-97, 2004.

[23] M. A. Jatoi, N. Kamel, A. S. Malik, I. Faye, and T. Begum, "Representing EEG source localization using finite element method," in Control System, Computing and Engineering (ICCSCE), 2013 IEEE International Conference on, 2013, pp. 168-172: IEEE.
[24] T. Nyoni and W. G. Bonga, "Neuromarketing Methodologies: More Brain Scans or Brain Scams?," 2017.

[25] R. D. Pascual-Marqui, "Discrete, 3D distributed, linear imaging methods of electric neuronal activity. Part 1: exact, zero error localization," arXiv preprint arXiv:0710.3341, 2007.

[26] J. J. Ermer, J. C. Mosher, S. Baillet, and R. M. Leahy, "Rapidly recomputable EEG forward models for realistic head shapes," Physics in Medicine \& Biology, vol. 46, no. 4, p. 1265, 2001.

[27] M. A. Jatoi, N. Kamel, I. Faye, A. S. Malik, J. M. Bornot, and T. Begum, "BEM based solution of forward problem for brain source estimation," in Signal and Image Processing Applications (ICSIPA), 2015 IEEE International Conference on, 2015, pp. 180-185: IEEE.

[28] F. Costa, H. Batatia, T. Oberlin, and J.-Y. Tourneret, "Skull Conductivity Estimation for EEG Source Localization," IEEE Signal Processing Letters, vol. 24, no. 4, pp. 422-426, 2017.

[29] B. Vanrumste, G. Van Hoey, R. Van de Walle, R. D. Michel, I. A. Lemahieu, and P. A. Boon, "Comparison of performance of spherical and realistic head models in dipole localization from noisy EEG," Medical Engineering and Physics, vol. 24, no. 6, pp. 403-418, 2002.

[30] R. Snieder and J. Trampert, "Inverse problems in geophysics," in Wavefield inversion: Springer, 1999, pp. 119-190.

[31] R. D. Pascual-Marqui, "Theory of the EEG inverse problem," Quantitative EEG analysis: methods and clinical applications, pp. 121140, 2009.

[32] L. Duque-Muñoz, J. Martinez-Vargas, G. Castellanos-Dominguez, J. Vargas-Bonilla, and J. López, "Non-linear Covariance Estimation for Reconstructing Neural Activity with MEG/EEG Data," in International Work-Conference on the Interplay Between Natural and Artificial Computation, 2017, pp. 334-344: Springer.

[33] J. C. Mosher and R. M. Leahy, "Recursive MUSIC: a framework for EEG and MEG source localization," IEEE Transactions on Biomedical Engineering, vol. 45, no. 11, pp. 1342-1354, 1998.

[34] S. Ikeda, R. Ishii, L. Canuet, and R. D. Pascual-Marqui, "Source estimation of epileptic activity using eLORETA kurtosis analysis," BMJ case reports, vol. 2017, pp. bcr-2017-222123, 2017.

[35] I. F. Gorodnitsky, J. S. George, and B. D. Rao, "Neuromagnetic source imaging with FOCUSS: a recursive weighted minimum norm algorithm," Electroencephalography and clinical Neurophysiology, vol. 95, no. 4, pp. 231-251, 1995.

[36] R. Khemakkhem, W. Zouch, A. Taleb-Ahmed, and A. B. Hamida, "A new combining approach to localizing the EEG activity in the brain: WMN and LORETA solution," in BioMedical Engineering and Informatics, 2008. BMEI 2008. International Conference on, 2008, vol. 1, pp. 821-824: IEEE.

[37] M. A. Jatoi, N. Kamel, A. S. Malik, I. J. A. p. Faye, and e. s. i. medicine, "EEG based brain source localization comparison of sLORETA and eLORETA," vol. 37, no. 4, pp. 713-721, 2014.

[38] M. S. Hamalainen, "Interpreting measured magnetic fields of the brain: estimates of current distributions," Helsinki Univ. of Technol., Rep, 1984

[39] R. D. Pascual-Marqui, C. M. Michel, and D. Lehmann, "Low resolution electromagnetic tomography: a new method for localizing electrical activity in the brain," International Journal of psychophysiology, vol. 18, no. 1, pp. 49-65, 1994.

[40] R. D. Pascual-Marqui, "Standardized low-resolution brain electromagnetic tomography (sLORETA): technical details," Methods Find Exp Clin Pharmacol, vol. 24, no. Suppl D, pp. 5-12, 2002.

[41] H. S. Liu, F. Yang, X. Gao, and S. Gao, "Shrinking LORETA-FOCUSS: a recursive approach to estimating high spatial resolution electrical activity in the brain," in Neural Engineering, 2003. Conference Proceedings. First International IEEE EMBS Conference on, 2003, pp. 545-548: IEEE.

[42] J. C. Mosher and R. M. Leahy, "Source localization using recursively applied and projected (RAP) MUSIC," IEEE Transactions on signal processing, vol. 47, no. 2, pp. 332-340, 1999.

[43] A.-S. Hincapié et al., "The impact of MEG source reconstruction method on source-space connectivity estimation: a comparison between 
minimum-norm solution and beamforming," NeuroImage, vol. 156, pp. 29-42, 2017.

[44] Y. Zhang, Y. Wang, J. Jin, and X. Wang, "Sparse Bayesian learning for obtaining sparsity of EEG frequency bands based feature vectors in motor imagery classification," International journal of neural systems, vol. 27, no. 02, p. 1650032, 2017.

[45] M. Dashti and A. M. Stuart, "The Bayesian approach to inverse problems," Handbook of Uncertainty Quantification, pp. 311-428, 2017.

[46] F. Costa, H. Batatia, T. Oberlin, C. D'giano, and J.-Y. Tourneret, "Bayesian EEG source localization using a structured sparsity prior," NeuroImage, vol. 144, pp. 142-152, 2017.

[47] Y. Bekhti, F. Lucka, J. Salmon, and A. Gramfort, "A hierarchical Bayesian perspective on majorization-minimization for non-convex sparse regression: application to M/EEG source imaging," arXiv preprint arXiv: $1710.08747,2017$.

[48] W. D. Penny, K. J. Friston, J. T. Ashburner, S. J. Kiebel, and T. E. Nichols, Statistical parametric mapping: the analysis of functional brain images. Elsevier, 2011.

[49] J. López, V. Litvak, J. Espinosa, K. Friston, and G. R. Barnes, "Algorithmic procedures for Bayesian MEG/EEG source reconstruction in SPM," Neurolmage, vol. 84, pp. 476-487, 2014.

[50] K. Friston et al., "Multiple sparse priors for the M/EEG inverse problem," NeuroImage, vol. 39, no. 3, pp. 1104-1120, 2008.

[51] D. Wipf and S. Nagarajan, "A unified Bayesian framework for MEG/EEG source imaging," NeuroImage, vol. 44, no. 3, pp. 947-966, 2009.

[52] S. Hu, D. Yao, and P. A. Valdes-Sosa, "Unified Bayesian estimator of EEG reference at infinity: rREST," arXiv preprint arXiv:1802.02268, 2018.
[53] K. Sekihara and S. S. Nagarajan, "A unified Bayesian framework for MEG/EEG source imaging," in Electromagnetic Brain Imaging: Springer, 2015, pp. 119-137.

[54] F. Goksu, N. F. Ince, and A. H. Tewfik, "Sparse common spatial patterns in brain computer interface applications," in Acoustics, Speech and Signal Processing (ICASSP), 2011 IEEE International Conference on, 2011, pp. 533-536: IEEE.

[55] D. Meunier, S. Achard, A. Morcom, and E. Bullmore, "Age-related changes in modular organization of human brain functional networks," Neuroimage, vol. 44, no. 3, pp. 715-723, 2009.

[56] K. Friston et al., "Bayesian decoding of brain images," Neuroimage, vol. 39, no. 1, pp. 181-205, 2008.

[57] J. Lopez, G. R. Barnes, and J. Espinosa Oviedo, Single MEG/EEG source reconstruction with multiple sparse priors and variable patches. 2012, pp. 136-144.

[58] M.-a. Sato et al., "Hierarchical Bayesian estimation for MEG inverse problem," NeuroImage, vol. 23, no. 3, pp. 806-826, 2004.

[59] Y. Yousra, F. Lucka, J. Salmon, and A. Gramfort, "A hierarchical Bayesian perspective on majorization-minimization for non-convex sparse regression: Application to $\mathrm{M} / \mathrm{EEG}$ source imaging," arXiv. org ePrint archive, 2017.

[60] S. Villena, M. Vega, S. D. Babacan, R. Molina, and A. K. Katsaggelos, "Bayesian combination of sparse and non-sparse priors in image super resolution," Digital Signal Processing, vol. 23, no. 2, pp. 530-541, 2013.

[61] J. López, F. Valencia, G. Flandin, W. Penny, and G. Barnes, "Reconstructing anatomy from electro-physiological data," NeuroImage, vol. 163, pp. 480-486, 2017.

[62] J. C. Ye, J. M. Kim, and Y. Bresler, "Subspace penalized sparse learning for joint sparse recovery," in Acoustics, Speech and Signal Processing (ICASSP), 2013 IEEE International Conference on, 2013, pp. 60396042: IEEE. 